\title{
The usefulness of Poynting's theorem in magnetic turbulence
}

\author{
Rudolf A. Treumann ${ }^{1, \mathrm{a}}$ and Wolfgang Baumjohann ${ }^{2}$ \\ ${ }^{1}$ Department of Geophysics and Environmental Sciences, Munich University, Munich, Germany \\ ${ }^{2}$ Space Research Institute, Austrian Academy of Sciences, Graz, Austria \\ ${ }^{\mathrm{a}}$ visiting scientist at: the International Space Science Institute, Bern, Switzerland
}

Correspondence: Wolfgang Baumjohann (wolfgang.baumjohann@oeaw.ac.at)

Received: 7 September 2017 - Revised: 21 November 2017 - Accepted: 29 November 2017 - Published: 15 December 2017

\begin{abstract}
We rewrite Poynting's theorem, already used in a previous publication (Treumann and Baumjohann, 2017a) to derive relations between the turbulent magnetic and electric power spectral densities, to make explicit where the mechanical contributions enter. We then make explicit use of the relativistic transformation of the turbulent electric fluctuations to obtain expressions which depend only on the magnetic and velocity fluctuations. Any electric fluctuations play just an intermediate role. Equations are constructed for the turbulent conductivity spectrum in Alfvénic and non-Alfvénic turbulence in extension of the results in the above citation. An observation-based discussion of their use in application to solar wind turbulence is given. The inertial range solar wind turbulence exhibits signs of chaos and self-organization.
\end{abstract}

Keywords. Space plasma physics (kinetic and MHD theory; turbulence)

\section{Introduction}

In a recent communication (Treumann and Baumjohann, 2017a) we used Poynting's theorem in electrodynamics in order to construct an experimentally accessible expression for the spectral energy density of the electromagnetic field in collisionless magnetic turbulence. That attempt turned out much simpler and therefore also more effective than our previous fairly involved inverse scattering theory (Treumann and Baumjohann, 2016) of electromagnetic fluctuations in magnetic turbulence. Since we used only electromagnetic theory, not referring to any mechanical fluid turbulence, it remained unclear to what extent an approach in turbulence like that one was justified. Magnetic turbulence at low frequencies - scales longer than the electron gyro-radius - involves both the electromagnetic and mechanical flow fields. Restric- tion to one of these components only apparently neglects an important part of the turbulence. This argument also applies to any experiments which use just measurements of magnetic fluctuations, calculate spectral energy densities, and possibly do not refer to electric field or velocity fluctuations. Determination of the power law shape of those spectra contains information about the turbulence, but its physical content remains inaccessible. Spectral slopes are sensitive to varying physical conditions (Treumann et al., 2015). Small changes in the slope, which within experimental errors are difficult to detect, may indicate completely different physics.

Observations of magnetic turbulence in the solar wind take advantage of their easy accessibility in order to determine spectral slopes of the turbulent magnetic energy densities (cf. e.g. Goldstein et al., 1995; Zhou et al., 2004, for early reviews) in the frequency domain. They enable us to distinguish between Kolmogorov's (Kolmogorov, 1941, 1962) spectral ranges of energy injection, constant energy flux, and dissipation in frequency space (cf. e.g. Alexandrova et al., 2009; Brown et al., 2015; Horbury et al., 2012; Sahraoui et al., 2009, 2012, 2013; Wicks et al., 2012, and references therein). Sometimes they enable distinction between Kolmogorov and Kraichnan regimes. They also provide absolute values of the turbulent magnetic energy density. Applying the Taylor hypothesis, limited information about the corresponding spatial scales has been obtained and, in a few cases, spectra of the electric field (Bale et al., 2005; Chen et al., 2011) and streaming velocity fluctuations (Podesta et al., 2006, 2007, 2010; Roberts, 2010; Podesta, 2011a, b; Šafránková et al., 2013, 2016) have been added. Measurements of turbulent density fluctuations in the solar wind (Celnikier et al., 1983; Chandran et al., 2009; Chen et al., 2012; Šafránková et al., 2013, 2016) have also been published. 
In the present note, following our previous attempt, Poynting's theorem is briefly re-examined in order to relate it to the inclusion of the mechanical part of turbulence and to clarify the effect of the electric and velocity fluctuations.

\section{Poynting's theorem in magnetic turbulence}

Measurement of the Poynting flux in order to infer the plasma wave energy flow in near-Earth space has a long history. One of the first attempts (LaBelle and Treumann, 1992) was to determine its direction and absolute value in plasmaspheric electromagnetic ion-cyclotron waves. More recently it was used to detect dispersive whistlers in Earth's bow shock (Sundqvist et al., 2012) which are expected to contribute to shock reformation in quasi-perpendicular shocks (cf. e.g. Balogh and Treumann, 2013, for a rather complete account) and to the investigation of the energy flow in kinetic Alfvén waves near the plasma sheet boundary (Stawarz et al., 2017) as a source of the auroral energy flow which often is attributed to the inflow of kinetic Alfvén waves (cf. e.g. Chaston et al., 2003) causing particle acceleration and radio emission (LaBelle and Treumann, 2002). These works deal with the Poynting flux in particular waves only.

In magnetic/magnetohydrodynamic turbulence (at nonrelativistic speeds) the equation of energy conservation, which is the generalization of Poynting's theorem in electrodynamics to the inclusion of mechanical energy transport, is quite generally written (Landau et al., 1998) in the form

$$
\frac{\partial}{\partial t}\left(\frac{1}{2} \rho v^{2}+\rho \varepsilon+\frac{B^{2}}{2 \mu_{0}}\right)=-\nabla \cdot \boldsymbol{q} .
$$

The vector $\boldsymbol{q}$ is the energy flux density, $\rho$ is the plasma mass density, $v$ is the velocity, and $\varepsilon=w-P / \rho$ is the internal energy, with $w$ the internal enthalpy and $P=\operatorname{tr} \mathbf{P} \equiv \frac{1}{3} P_{i i}$ the (scalar) pressure, and the relativistically small electric field density has been suppressed in the time-derivative term on the left. In this form the energy law accounts for all the energy in the turbulence. The energy flux vector $\boldsymbol{q}$ contains all the dissipative processes, mechanical and electromagnetic, in particular all anomalous processes which contribute to dissipation. The former (mechanical) terms contain a mechanical dissipation tensor, with bulk and shear viscosity coefficients. The latter (electromagnetic) terms are inherent to a conductivity tensor $\sigma_{i j}$, which enters Ohm's law and which can always be written in its simplest form, such that the current is given by $J_{i}=\sigma_{i j} E_{j}$, where $\boldsymbol{E}=-\boldsymbol{v} \times \boldsymbol{B}$ is the (relativistically correct) electric field. For finite electrical resistance, the relation between the electric field and current $\boldsymbol{J}$ becomes $\boldsymbol{E}+\boldsymbol{v} \times \boldsymbol{B}=\boldsymbol{\sigma}^{-1} \cdot \boldsymbol{J}$, an expression which is general in the sense that the various dissipative processes contributing to this generalized Ohm's law (cf. e.g. Baumjohann and Treumann, 1996; Krall and Trivelpiece, 1973) are included in the definition of the conductivity tensor $\sigma$ which in all realistic cases, if made explicit, becomes an involved expres- sion. Any dissipation of electromagnetic energy is given by the product $-\boldsymbol{E} \cdot \boldsymbol{J}$. Neglecting collisional dissipation, as is usually done in ordinary MHD, one has

$$
\boldsymbol{q}=\rho \boldsymbol{v}\left(\frac{1}{2} v^{2}+w\right)+\frac{1}{\mu_{0}} \boldsymbol{B} \times(\boldsymbol{v} \times \boldsymbol{B})
$$

Written in terms of the electromagnetic field, energy conservation takes the form

$$
\frac{\partial}{\partial t} \frac{B^{2}}{2 \mu_{0}}=-\frac{1}{\mu_{0}} \nabla \cdot(\boldsymbol{E} \times \boldsymbol{B})-\nabla \cdot \boldsymbol{q}_{m}-\frac{\partial}{\partial t} \mathcal{E}_{m}
$$

with

$\boldsymbol{q}_{m}=\rho \boldsymbol{v}\left(\frac{1}{2} v^{2}+w\right), \quad \mathcal{E}_{m}=\frac{1}{2} \rho v^{2}+\rho \varepsilon$.

Any possibly occurring dissipation is solely due to turbulent mixing and in this sense is "anomalous". This is Poynting's theorem completed with the two mechanical terms on the right. On the left is the time variation of the magnetic energy density. The first term on the right is the divergence of the electromagnetic energy flux vector, a familiar quantity. The other two terms, depending on their signs, either pump energy into the magnetic field by mechanical motion, as in the case of a dynamo, or dissipate magnetic energy.

Since any dissipation of magnetic energy, either positive or negative, can always be written as the above product $-\boldsymbol{E} \cdot \boldsymbol{J}$, Poynting's theorem for the electromagnetic field under ideal dissipationless conditions in magnetic/magnetohydrodynamic turbulence can be written as

$\frac{\partial}{\partial t} \frac{B^{2}}{2 \mu_{0}}=-\boldsymbol{E} \cdot \boldsymbol{J}-\frac{1}{\mu_{0}} \nabla \cdot(\boldsymbol{E} \times \boldsymbol{B})$,

which is its familiar version in electrodynamics, and

$\boldsymbol{E} \cdot \boldsymbol{J}=\nabla \cdot \boldsymbol{q}_{m}+\frac{\partial}{\partial t} \mathcal{E}_{m}+\boldsymbol{E} \cdot \boldsymbol{\sigma}_{\mathrm{an}}^{-1} \cdot \boldsymbol{E}$.

A possibly present anomalous conductivity $\sigma_{\text {an }}$ caused by kinetic processes on scales shorter than the ion or electron inertial lengths or gyroradii $<\lambda_{i, e}, r_{\mathrm{ci}, \text { ce }}$ would appear as the last term in Eq. (6), but is not explicitly considered in the following. In collisionless and non-viscous turbulent plasmas the latter form applies at scales exceeding the Debye length and is far away from any molecular scale to which dissipation of the turbulent mechanical energy is attributed. In contrast, the turbulent electromagnetic energy is ultimately dissipated at least already at electron scales $<\lambda_{e}, r_{\mathrm{ce}}$ by spontaneous reconnection (Treumann et al., 2015) in small-scale current filaments. ${ }^{1}$ These are generated progressively by turbulent self-organization in the spectral energy flow (Treumann and Baumjohann, 2017a) towards the short scales. There dissipation is anomalous, mediated by plasma-kinetic processes.

\footnotetext{
${ }^{1}$ At scales shorter than the electron gyroradius, electrons demagnetize and no longer contribute to magnetic fluctuations, electron thermal pressure does not balance the Lorentz force which contracts the current, and collisionless reconnection is spontaneous and ex-
} 


\section{Application to turbulent fluctuations}

Writing all quantities as sums of mean fields plus fluctuations $F=\bar{F}+\delta F$ with average $\bar{F}=\bar{F}$ and $\overline{\delta F}=0$ in Eq. (5), averaging, subtracting the scale-averaged equation, and dropping the averaged products of the fluctuations as these depend only on the mean-field scale, we find

$$
\begin{aligned}
& \frac{\partial}{\partial t}\left(\frac{2 \overline{\boldsymbol{B}} \cdot \delta \boldsymbol{B}}{2 \mu_{0}}+\frac{(\delta B)^{2}}{2 \mu_{0}}\right) \\
& \quad=-\delta \boldsymbol{E} \cdot \overline{\boldsymbol{J}}-\delta \boldsymbol{E} \cdot \delta \boldsymbol{J}-\frac{1}{\mu_{0}} \nabla \cdot[\delta \boldsymbol{E} \times \overline{\boldsymbol{B}}+\delta \boldsymbol{E} \times \delta \boldsymbol{B}]
\end{aligned}
$$

with mean electric field $\overline{\boldsymbol{E}}=0$ (see the next section below), and defining $\delta \boldsymbol{J}=\boldsymbol{\sigma}^{T} \cdot \delta \boldsymbol{E}$, where $\boldsymbol{\sigma}^{T}$ is an equivalent turbulent conductivity tensor chosen such that when it relates the turbulent current to the turbulent electric field, the mean current vanishes. The result is

$$
\begin{aligned}
& \frac{\partial}{\partial t}\left(\frac{2 \overline{\boldsymbol{B}} \cdot \delta \boldsymbol{B}}{2 \mu_{0}}+\frac{(\delta B)^{2}}{2 \mu_{0}}\right) \\
& \quad=-\delta \boldsymbol{E} \cdot \boldsymbol{\sigma}^{T} \cdot \delta \boldsymbol{E}-\frac{1}{\mu_{0}} \nabla \cdot[\delta \boldsymbol{E} \times \overline{\boldsymbol{B}}+\delta \boldsymbol{E} \times \delta \boldsymbol{B}],
\end{aligned}
$$

which is the basic equation used in Treumann and Baumjohann (2017a). Restricting it to magnetically non-compressive turbulence $\delta \boldsymbol{B} \cdot \overline{\boldsymbol{B}}=0$ makes the first term on the left vanish. The first term in the brackets on the right vanishes for $\delta \boldsymbol{E} \| \overline{\boldsymbol{B}}$, the case $\boldsymbol{k} \perp \overline{\boldsymbol{B}}$ of propagation of the turbulent fluctuations perpendicular to the mean field. For parallel propagation this term contains the excluded compressive magnetic component. We are thus left with the simplified Poynting equation

$$
\frac{\partial}{\partial t} \frac{\left(\delta B_{\perp}\right)^{2}}{2 \mu_{0}}=-\delta \boldsymbol{E} \cdot \boldsymbol{\sigma}^{T} \cdot \delta \boldsymbol{E}-\frac{1}{\mu_{0}} \nabla \cdot\left[\delta \boldsymbol{E} \times \delta \boldsymbol{B}_{\perp}\right] .
$$

All dynamics of the turbulent mechanical flow is implicit in $\boldsymbol{\sigma}^{T}$, which (keeping an anomalous conductivity $\boldsymbol{\sigma}_{\text {an }}$ ) is formally defined as

$\boldsymbol{\sigma}^{T}=(\delta \boldsymbol{E})^{-1} \cdot\left[\boldsymbol{\sigma}_{\text {an }}+\nabla \cdot \delta \boldsymbol{q}_{m}+\frac{\partial}{\partial t} \delta \mathcal{E}_{m}\right] \cdot(\delta \boldsymbol{E})^{-1}$

where $\delta \boldsymbol{q}_{m}, \delta \mathcal{E}_{m}$ are the fluctuations of $\boldsymbol{q}_{m}, \mathcal{E}_{m}$. Once, by the means of measuring the electromagnetic turbulent fluctuation spectrum, the turbulent conductivity spectrum $\boldsymbol{\sigma}_{\omega \boldsymbol{k}}^{T}$ has been determined as a function of fluctuation frequency $\omega$ and wavenumber $\boldsymbol{k}$, its transformation back into real space provides a relation to the turbulent mechanical quantities.

plosive, causing electron exhausts, strongly deformed electron distributions, and electron beams. Dissipation here is kinetically and electrostatically provided by plasma waves (Langmuir, ion sound, Bernstein, electron holes). Except for a possible filamentary Weibel mode which causes further filamentation of the current and turbulence, no non-radiative magnetic fields are generated here. Hence, the magnetic turbulence spectrum should decay at those scales. High-frequency and thus weak-radiative fields can be produced in addition by the electron cyclotron maser instability inside the exhaust.

\section{Turbulent electric and velocity fields}

A difficulty arises in dealing with the electric field. Relativistic invariance requires its transformation into the rest frame of the flow $\boldsymbol{E}^{\prime}=\boldsymbol{E}+\boldsymbol{v} \times \boldsymbol{B}$. In an ideal turbulent medium the moving frame speed depends on the fluctuation scale, which in general makes it difficult (if not impossible) to define a common moving frame valid on all scales. Splitting into mean and fluctuating quantities yields the averaged field

$\overline{\boldsymbol{E}^{\prime}}=\overline{\boldsymbol{E}}+\overline{\boldsymbol{v}} \times \overline{\boldsymbol{B}}+\overline{\delta \boldsymbol{v} \times \delta \boldsymbol{B}}$,

which in the moving frame must vanish. This gives the mean electric field $\overline{\boldsymbol{E}}=-\overline{\boldsymbol{v}} \times \overline{\boldsymbol{B}}-\overline{\delta \boldsymbol{v} \times \delta \boldsymbol{B}}$. Measurement of the velocity fluctuations $\delta \boldsymbol{v}$ in the scale range of interest is required in the averaged second term. The fluctuating primed electric field becomes

$\delta \boldsymbol{E}^{\prime}=\delta \boldsymbol{E}+\delta \boldsymbol{v} \times \overline{\boldsymbol{B}}+\delta \boldsymbol{v} \times \delta \boldsymbol{B}+\overline{\boldsymbol{v}} \times \delta \boldsymbol{B}-\overline{\delta \boldsymbol{v} \times \delta \boldsymbol{B}}$.

The mean magnetic field $\overline{\boldsymbol{B}}$ and the last averaged term are constant on the fluctuation scale. In an infinitely extended medium without boundaries the last term can be dropped, yielding

$\delta \boldsymbol{E}=-\delta \boldsymbol{v} \times \overline{\boldsymbol{B}}-\delta \boldsymbol{v} \times \delta \boldsymbol{B}-\overline{\boldsymbol{v}} \times \delta \boldsymbol{B}$,

which is to be used in Eq. (9). It requires knowledge of the velocity fluctuations on the same scales (and with same resolution) as the magnetic fluctuations. The second term on the right measures the "alignment" of the magnetic and velocity fluctuations.

In so-called purely Alfvénic turbulence, $\delta \boldsymbol{v} \| \delta \boldsymbol{B}$ and the cross-helicity (normalized to the total energy) is close to unity, resulting in a linear relation for the fluctuating electric field $\delta \boldsymbol{E}=-\delta \boldsymbol{v} \times \overline{\boldsymbol{B}}-\overline{\boldsymbol{v}} \times \delta \boldsymbol{B}$. The electric fluctuations are perpendicular to both $\delta \boldsymbol{B}, \delta \boldsymbol{v}$ in this case. The Poynting flux vector term in Eq. (9) assumes the form $-\overline{\boldsymbol{B}} \cdot \nabla\left(\delta \boldsymbol{v} \cdot \delta \boldsymbol{B}_{\perp}\right) / \mu_{0}$, which eliminates the electric fluctuations in favour of the velocity field and reduces Eq. (9) to

$$
\begin{gathered}
\frac{\partial}{\partial t} \frac{\left(\delta B_{\perp}\right)^{2}}{2 \mu_{0}}=-\sigma_{\perp}^{T} \bar{B}^{2}(\delta \boldsymbol{v})^{2}-\frac{1}{\mu_{0}} \overline{\boldsymbol{B}} \cdot \nabla\left(\delta \boldsymbol{v} \cdot \delta \boldsymbol{B}_{\perp}\right) \\
-\sigma_{\perp}^{T}\left[\bar{v}^{2}\left(\delta \boldsymbol{B}_{\perp}\right)^{2}-\left(\overline{\boldsymbol{v}} \cdot \delta \boldsymbol{B}_{\perp}\right)^{2}\right]
\end{gathered}
$$

where $\sigma_{\perp}^{T}$ is the turbulent conductivity parallel to $\delta \boldsymbol{E}$, i.e. perpendicular to both $\delta \boldsymbol{B}_{\perp}$ and $\overline{\boldsymbol{B}}$. The last terms contain only the mean flow components $\overline{\boldsymbol{v}}^{\perp}$ perpendicular to $\delta \boldsymbol{B}_{\perp}$. The complications they introduce disappear when transforming to the easily determined mean flow $\bar{v}=0$. The Poynting term vanishes when considering spatial dependencies perpendicular to the mean field. More generally, since in Alfvénic turbulence $\delta \boldsymbol{v}=\alpha \delta \boldsymbol{B}_{\perp}$ with $\alpha$ some angulardependent scalar factor (which can, in principle, be determined from the fluctuations), the argument of the Poynting 
vector can be expressed by $\left(\delta B_{\perp}\right)^{2}$. Except for any spatial dependence of $\alpha$, the magnetic and velocity fluctuation spectra should thus be comparable in Alfvénic turbulence for either parallel or perpendicular propagation. (One may note that for cross-helicity $\delta \boldsymbol{v} \cdot \delta \boldsymbol{B}_{\perp} /\left|\delta \boldsymbol{v} \cdot \delta \boldsymbol{B}_{\perp}\right| \approx \pm 1$ the second term on the right in Eq. (14) disappears.)

Fourier transforming in space and time in the infinitely extended domain, assuming stationary and homogeneous conditions and constant $\alpha$ yields

$\sigma_{\perp \omega k}^{T}=\frac{i \omega}{2 \mu_{0} \bar{B}^{2}}\left(1-2 \alpha \frac{\boldsymbol{k} \cdot \overline{\boldsymbol{B}}}{\omega}\right) \frac{\left(\delta B_{\perp}\right)_{\omega \boldsymbol{k}}^{2}}{\left(\delta v_{\perp}\right)_{\omega \boldsymbol{k}}^{2}}$

This holds in Alfvénic turbulence. (The contribution of a finite mean speed may be retained any time when wanted.) For cross-helicity one, the expression in parentheses in the first term on the right reduces to unity. ${ }^{2}$ The turbulent response of the plasma contained in the conductivity spectrum $\boldsymbol{\sigma}_{\perp \omega \boldsymbol{k}}^{T}$ is, under stationary and homogeneous conditions, given by the ratio of the spectral energy densities of the turbulent magnetic and velocity fields. (We note in passing that this expression can also be exploited when constructing (Treumann and Baumjohann, 2017a) a low-frequency "turbulent dispersion relation" $\mathcal{N}^{2} \equiv k^{2} c^{2} / \omega^{2}=i \sigma_{\perp \omega k}^{T} / \omega \epsilon_{0}$, which is not the solution of a linear eigenmode problem, but determines the nonlinear relation between the turbulent frequencies $\omega$ and wavenumbers $\boldsymbol{k}$.)

For non-Alfvénic turbulence $\delta \boldsymbol{v} \perp \delta \boldsymbol{B}$, i.e. $\delta \boldsymbol{v} \cdot \delta \boldsymbol{B}=0$, which means that the cross-helicity vanishes. It is convenient to distinguish velocity fluctuations parallel and perpendicular to the mean field. If $\delta \boldsymbol{v} \| \overline{\boldsymbol{B}}$, the turbulent electric, magnetic, and velocity fluctuations form a mutually orthogonal system $\delta \boldsymbol{E}=-\delta \boldsymbol{v} \times \delta \boldsymbol{B}_{\perp}$. Hence Poynting's vector becomes $\delta \boldsymbol{E} \times \delta \boldsymbol{B}_{\perp}=\delta \boldsymbol{v}_{\|}\left(\delta B_{\perp}\right)^{\frac{1}{2}}$, giving from (9)

$\frac{\partial}{\partial t} \frac{\left(\delta B_{\perp}\right)^{2}}{2 \mu_{0}}=-\sigma_{\perp}^{T}\left(\delta B_{\perp}\right)^{2}\left(\delta v_{\|}\right)^{2}-\frac{1}{\mu_{0}} \nabla_{\|}\left[\delta v_{\|}\left(\delta B_{\perp}\right)^{2}\right]$

for non-compressive non-Alfvénic magnetic turbulence. It is obvious that in this case the cross-helicity contributes through the (parallel) divergence of the Poynting flux. Unlike the Alfvénic case, the last term in the above expression generally cannot be reduced further. Moreover, the first term on the right is a triple product, which makes any further treatment difficult.

If the turbulence is independent in the parallel direction such that the parallel turbulent wave vectors $k_{\|}=0$ vanish, then the last equation simplifies and can be solved for the

\footnotetext{
${ }^{2}$ There is, of course, no obvious reason for $\alpha$ to be constant. In general it will depend on space and time, which is suggested by the radial variation of the solar wind spectra with increasing solar distance (Roberts, 2010). Locally, the assumption of constancy is well justified, however, as is also confirmed by solar wind observations at $1 \mathrm{AU}$ of the constancy of the cross-helicity (Podesta et al., 2010).
}

perpendicular non-Alfvénic conductivity spectrum:

$\sigma_{\perp \omega \boldsymbol{k}_{\perp}}^{T}=\frac{i \omega}{2 \mu_{0}}\left[\log \left(\delta B_{\perp}\right)^{2}\right]_{\omega \boldsymbol{k}_{\perp}}\left[\left(\delta v_{\|}\right)_{\omega \boldsymbol{k}_{\perp}}^{2}\right]^{-1}$

The logarithmic dependence on the spectral energy density of the magnetic turbulence implies that the conductivity spectrum is mainly determined by the spectral energy density in the turbulence of the mechanical flow. This is also the case when $k_{\|} \neq 0$, because then the above equation can be brought into the form

$\left(\frac{\partial}{\partial t}+2 \delta v_{\|} \nabla_{\|}\right) \frac{\log \left(\delta B_{\perp}\right)^{2}}{2 \mu_{0}}=-\frac{1}{\mu_{0}} \nabla_{\|} \delta v_{\|}-\sigma_{\perp}^{T}\left(\delta v_{\|}\right)^{2}$

where the dependence on the magnetic fluctuation spectrum remains logarithmic as well. Again, in homogeneous stationary turbulence this can be reduced to an equation for the spectral density of $\sigma_{\perp}^{T}$.

Otherwise, for $\delta \boldsymbol{v} \perp \overline{\boldsymbol{B}}$, one has $\delta \boldsymbol{B} \| \overline{\boldsymbol{B}}$ as a consequence of $\delta \boldsymbol{v} \perp \delta \boldsymbol{B}$. We called this case compressive magnetic turbulence (Treumann and Baumjohann, 2017a) and, for our purposes, excluded it from consideration.

Further conclusions can be drawn when considering the propagation of the turbulent fluctuations. Propagation perpendicular to $\overline{\boldsymbol{B}}$ of magnetically non-compressive fluctuations $\left(\delta \boldsymbol{B}_{\|}=0\right)$ implies $\delta \boldsymbol{E} \| \overline{\boldsymbol{B}}$. Hence the first term on the right in Eq. (13) is zero, and since the magnetic and electric fluctuation fields are orthogonal, lying both in the plane perpendicular to the mean field, one has $\delta \boldsymbol{v} \| \overline{\boldsymbol{B}}$, i.e. all velocity fluctuations which contribute are parallel to the mean field. Moreover, in Eq. (16) the last term on the right thus disappears and, after Fourier transformation, one obtains a simple expression for the turbulent conductivity spectrum in homogeneous stationary turbulence in this case (Treumann and Baumjohann, 2017a).

Any magnetically compressive turbulence $\delta \boldsymbol{B} \| \overline{\boldsymbol{B}}$, which so far has been excluded here, requires a separate investigation. In this case, still considering only electromagnetic fluctuations with $\delta \boldsymbol{E} \cdot \delta \boldsymbol{B}=0$, the electric fluctuations corresponding to $\delta \boldsymbol{B}_{\|}$are perpendicular to $\overline{\boldsymbol{B}}$, in agreement with Eq. (13). One obtains after some simple algebra that

$\delta \boldsymbol{E} \times \delta \boldsymbol{B}_{\|}=\delta b_{\|}\left(1+\delta b_{\|}\right) \bar{B}^{2} \delta \boldsymbol{v}_{\perp}^{\perp}$

where $\delta v_{\perp}^{\perp}$ is the velocity fluctuation perpendicular to the mean magnetic and turbulent electric fields, and $\delta b_{\|}=$ $\delta B_{\|} / \bar{B}$ is the ratio of the compressive amplitude of the magnetic fluctuations to the mean field. The divergence of this expression is the contribution of the compressive part of the magnetic turbulence. It vanishes for parallel propagation, contributing only for propagation $\boldsymbol{k}=\boldsymbol{k}_{\perp}$ perpendicular to the mean field. Combining all the terms produces the equa- 
tion

$$
\begin{aligned}
& \frac{\partial}{\partial t} \frac{\left(\delta b_{\|}\right)^{2}}{2 \mu_{0}}=-\sigma_{\|}^{T}\left(\delta v_{\perp}^{\perp}\right)^{2}\left[1+\left(\delta b_{\|}\right)^{2}\right] \\
& -\frac{1}{\mu_{0}} \nabla_{\perp} \cdot\left[\delta v_{\perp}^{\perp} \delta b_{\|}\left(1+\delta b_{\|}\right)\right]
\end{aligned}
$$

for the magnetically compressive component. Experimentally it is a simple matter to separate out $\delta \boldsymbol{B}_{\|}$. We do not invest further in any discussion of this case.

\section{Discussion and conclusions}

Poynting's theorem provides additional information about turbulence which so far had not been exploited. It allows us to account for the relativistic effect in the electric field and reduces it to a measurement of the turbulent velocity and magnetic fields as suggested by Eq. (13). This cannot be circumvented by no means. It is interesting to briefly discuss more recent measurements of electric field, velocity, and also density fluctuations (Bale et al., 2005; Chen et al., 2011; Podesta et al., 2006, 2007, 2010; Podesta, 2011a, b; Roberts, 2010) in this light.

The specifications of Sect. 4 show that, as expected from electrodynamics, replacing the electric fluctuations in electromagnetic turbulence, the magnetic and velocity fields become related. This follows from relativity. The electric fluctuation field plays an intermediate role of an mediator only. The versions of Poynting's theorem given above explicate the interrelation. They can be applied to stationary homogeneous turbulence providing expressions for the spectrum of the turbulent conductivity as a functional of the magnetic and velocity power spectral densities similar to those given previously (Treumann and Baumjohann, 2017a) but expressed here in terms of the velocity fields. There we insisted on the independent determination of the electric and magnetic power spectral densities. It turns out that determination of the spectrum of turbulent velocities on all scales is more important.

Observations in the solar wind on comparably large scales indicate that the velocity and magnetic spectra in the inertial MHD range exhibit different slopes (Podesta et al., 2006, 2007; Podesta, 2011a). Velocity power spectra are typically flatter, of slope $-\frac{3}{2}$ (2-D or Kraichnan), than magnetic spectra at $1 \mathrm{AU}$, which are close to the $3-\mathrm{D}-$ Kolmogorov $-\frac{5}{3}$ with apparently less power in the kinetic than magnetic energy fluctuations. In fact, there is no obvious reason why they should be similar. Any magnetic fluctuations $\delta \boldsymbol{B}$ are, through Ampère's law, related to fluctuations of the electric current

$\delta \boldsymbol{J}=e \bar{N}\left(\delta \boldsymbol{v}_{i}-\delta \boldsymbol{v}_{e}\right)+e \delta N\left(\overline{\boldsymbol{v}}_{i}-\overline{\boldsymbol{v}}_{e}\right)$

assuming quasi-neutrality in turbulence. Examples are diamagnetic currents in pressure gradients. Under stationary conditions this reduces to pressure balance. It is the difference in the fluctuations of the ion and electron velocities and the density fluctuations which both contribute. At long MHD scales the average velocities cancel and the last term in the current disappears, but in the first term the ion and electron velocity fluctuations are not aligned and contribute differently to the spectra. Measured fluctuations in the flow $\delta \boldsymbol{v}$ have little in common with the fluctuations of the current. At short scales the second term on the right in the current contributes through the density fluctuations which are caused mainly by fluctuations of the plasma pressure and thus are related to the transverse magnetic pressure. With increasing solar distance in the solar wind, the velocity spectra though in the inertial scale range, still being of lower spectral density than the magnetic spectra, seem to approach the Kolmogorov slope (Roberts, 2010) while at the same time intensifying. If confirmed, a simple explanation is that in solar wind turbulence the effect of decreasing magnetic field on the flow weakens with increasing solar distance, thus gradually losing dominance.

\subsection{Data-based thermodynamic considerations}

The above measurements of the turbulent solar wind velocity spectrum were restricted to the MHD frequency range $\lesssim 10^{-2} \mathrm{~Hz}$. More recent observations (Šafránková et al., 2013, 2016) based on a sophisticated technique aboard the Spektr-R spacecraft, extended to higher frequencies into the range $\lesssim 2 \mathrm{~Hz}$, presumably scale below the ion gyroradius, where ion kinetic effects become important, for instance in supporting kinetic Alfvén waves, and the ions demagnetize.

These measurements confirm the $\sim-\frac{3}{2}$ slope of the turbulent velocity spectrum in the MHD range at frequencies below the ion-cyclotron frequency (scales, presumably longer than the ion gyro and/or inertial scales), thus being more 2-D and flatter than those observed about Kolmogorov-turbulent magnetic spectra. At their higher frequencies they partially cover the kinetic non-magnetized ion range spectra and exhibit power laws of a steeper slope close to -3 , indicating that the turbulent (ion) velocity fluctuations enter a different, presumably still inertial fluid regime when decoupling from the magnetic field. Currents which contribute to the magnetic fluctuations here are carried by magnetized electrons either perpendicularly, as drift currents in the density and temperature gradients of the turbulent eddies, thereby forming narrow scale current filaments, or along the magnetic field as kinetic Alfvén waves (Alexandrova et al., 2013). Signatures of the proximity to this regime are visible as undulations in the velocity spectrum above say $3 \times 10^{-2} \mathrm{~Hz}$ already where they form a weak bump on the spectrum which is even more expressed in the density spectrum (first observed already by Celnikier et al., 1983, their Fig. 1) which, in general, does not follow either Kraichnan's or Kolmogorov's prescription.

It is also of interest that, in the inertial range, the temperature spectrum mimics the velocity spectrum (Šafránková et al., 2016). Thus inertial range kinetic energy $\mathrm{d} \epsilon$ and thermal energy $\mathrm{d} T$ follow each other. Assuming ideal gas conditions 
implies that

$\mathrm{d} \epsilon=c_{v} \mathrm{~d} T$.

Therefore, the specific heat $c_{v} \approx$ const (within the uncertainty of the measurements) does not change across the inertial range. Such processes are isentropic with

$T \sim N^{\gamma-1}$

where $\gamma=c_{p} / c_{v}$ is the ratio of specific heats. Using the average inertial range slopes (see Šafránková et al., 2016, Fig. 1), we then find from the general adiabatic (isentropic) equation (cf. e.g. Kittel and Kroemer, 1980)

$\mathrm{d} \log T-(\gamma-1) \mathrm{d} \log N=0$

that in the solar wind inertial range the ratio of specific heats as determined from the fluctuations in density and thermal speed (Šafránková et al., 2016) is $\gamma \approx 1.82$, which implies that under the ideal gas assumption one finds from the relation

$\gamma=\frac{2+D}{D}$

between $\gamma$ and the number of dimensions $D$ (cf. e.g. Kittel and Kroemer, 1980; Landau and Lifschitz, 1994) that the inertial range has fractal dimension $D \approx 2.46$, which again implies deterministic chaos, self-organization, and structure formation (cf. e.g. Barnsley, 1988; Eckmann and Ruelle, 1985; Eckmann and Procaccia, 1986; Zaslavsky, 1985) in this range. Since, at least in part of the inertial range, the density and magnetic spectra behave similarly, this reasoning also applies to the turbulent magnetic field.

Entering the ion-kinetic range at higher frequencies, the temperature adjusts to the steeper slope of $\sim-\frac{5}{2}$, suggesting non-adiabaticity and heating over the velocity spectrum, as is of course expected when ion-kinetic processes like heating by kinetic Alfvén wave turbulence take over in this range.

\subsection{Application to Alfvénic solar wind turbulence}

It would be desirable to apply the measurements published above to our theoretical determination of the conductivity spectrum. Unfortunately, however, the experimental spectral energy densities are available only in frequency space. Application of the Taylor hypothesis to transfer them into wavenumber space implies imposing a linear Galilean transformation relation $\omega=\overline{\boldsymbol{v}} \cdot \boldsymbol{k}$ which may hold for very high nonrelativistic average speeds (see also the brief discussion below) and thus in frequency-wavenumber space restricts one to multiplication of the conductivity spectrum with a Dirac function $\delta(\omega-\overline{\boldsymbol{v}} \cdot \boldsymbol{k})$. In the Alfvénic turbulence case one may formally obtain from the measurements of, say, Šafránková et al. (2016), and using Eq. (15) that

$\sigma_{\perp \omega \boldsymbol{k}} \sim\left(1-2 \bar{B} \alpha \frac{k_{\|}}{\omega}\right) \omega^{-\left(s_{\delta B}-s_{\delta v}-1\right)} \delta(\omega-\overline{\boldsymbol{v}} \cdot \boldsymbol{k})$ where $s_{\delta v}, s_{\delta B}$ are the respective experimental slopes of the velocity and magnetic field spectra. Since these are about $\sim$ $\frac{3}{2}$ and $\sim \frac{5}{3}$ respectively, the conductivity spectrum in the inertial range is also the power law of the index (up to the factor in brackets and the Dirac function) $s_{\sigma}=s_{\delta B}-s_{\delta v}-1 \approx-\frac{5}{6}$, indicating an increase in conductivity $\sigma_{\perp \omega \boldsymbol{k}} \sim \omega^{5 / 6} \delta(\omega-\overline{\boldsymbol{v}}$. $\boldsymbol{k}$ ) with frequency (shrinking temporal scale). Applying the Dirac function which the Taylor hypothesis in addition imposes yields the wavenumber dependence

$\sigma_{\perp \boldsymbol{k}} \sim\left(1-\alpha \bar{B} \frac{k_{\|}}{\overline{\boldsymbol{v}} \cdot \boldsymbol{k}}\right)(\overline{\boldsymbol{v}} \cdot \boldsymbol{k})^{\frac{5}{6}}$

(Note that $k_{\|}$refers to the mean magnetic field, while in the denominator the wavenumber is parallel to the average flow through Taylor's hypothesis which artificially reintroduces $\overline{\boldsymbol{v}}$ at this late place after developing the theory!) If this finding is confirmed and applies, the inertial range turbulent resistance drops in frequency and wavenumber, meaning that the inertial range in Alfvénic turbulence behaves increasingly less dissipative towards shorter scales. The system is collisionless, so this contradicts the expected self-organization and structure formation (formation of progressively shorter scale current filaments, eddies, etc.) which we have inferred above from fundamental thermodynamic arguments without making any reference to any additional hypothesis. This should not be the case. So this result may provide a strong argument against the application of the Taylor hypothesis, at least at short scales, i.e. large wavenumbers and frequencies. For the above-mentioned reasons concerning observations, such a conclusion must, however, be drawn with care.

At this point a general remark on the use of Taylor's hypothesis is in order. It not only reduces the wavenumberfrequency spectrum to the inclusion of a delta function, but it also reduces the "turbulent dispersion relation" to a linear relation. This might indeed hold as long as the flow velocity is very high, $|\overline{\boldsymbol{v}}| \gg \sup |\delta \boldsymbol{v}|$, a trivial condition. Instead, the "correct" turbulent dispersion relation for magnetic turbulence is given through the frequency-wavenumber spectrum of the turbulent conductivity (see Treumann and Baumjohann, 2017a). In addition, the Taylor hypothesis applies only to turbulent structures which propagate along the mean flow such that $\boldsymbol{k} \| \overline{\boldsymbol{v}}$. Any turbulence propagating at an angle, for instance the rotational velocity component of a turbulent eddy, is thus affected only up to an angle where the projection of the mean speed of the flow onto the wavenumber vector still by far exceeds the turbulent speed. Any strictly perpendicular wave is not affected by Taylor's hypothesis and thus principally cannot become transformed into wavenumber space.

The observations used above make no difference between the propagation directions. Thus any distinction is impossible and any application of spatial scales like gyroradii and inertial scales is questionable because it applies only to part of the mixture of components which makes up the spectra. In order to solve this problem, observations should be split into 
components perpendicular and parallel to $\bar{v}$ and the Taylor hypothesis should be applied to the parallel component only.

\section{Conclusions}

In the previous section we applied Poynting's theorem to derive expressions between the turbulent conductivity and measurable spectral energy densities. These expressions are formulated in terms of the magnetic and velocity spectra. The electric field appears just on an intermediate step, becoming eliminated by the relativistic transformation. These expressions may be useful in application to observations, but require precise measurements of the velocity field fluctuations. This is the main experimental difficulty. Their knowledge is of general interest in turbulence theory as they allow construction of a turbulent dispersion relation which is not a solution of an eigenmode equation but determines the relation between observed frequencies and wavenumbers. This should provide a useful experimental input into the conventional approach to both fully developed strong (Zhou et al., 2004; Zank et al., 2012) and weak (Yoon, 2007; Yoon and Fang, 2007; Boldyrev and Perez, 2009) stationary and homogeneous magnetohydrodynamic turbulence.

Finally we note that we did not use Elsasser (Elsasser, 1950) variables here, the mixed magnetic and flow fields which are usually used in magnetohydrodynamic turbulence theory (Biskamp, 2003; Zhou et al., 2004; Zank et al., 2012). Reformulation of the results in these variables is a simple matter. This will be left for a separate investigation.

Data availability. No data sets were used in this article.

Competing interests. The authors declare that they have no conflict of interest.

Acknowledgement. This work was part of a Visiting Scientist Programme in 2007 at the International Space Science Institute Bern. We acknowledge the interest of the ISSI directorate and the friendly hospitality of the ISSI staff. We thank the ISSI technical administrator Saliba F. Saliba for help, and the librarians Andrea Fischer and Irmela Schweitzer for access to the library and literature. We thank the anonymous reviewer for the constructive critical comments and for directing our attention to some recent publications on measurements of solar wind turbulent velocity and density spectra.

The topical editor, Elias Roussos, thanks one anonymous referee for help in evaluating this paper.

\section{References}

Alexandrova, O., Saur, J., Lacombe, C., Mangeney, A., Mitchell, J., Schwartz, S. J., and Robert, P.: Universality of solar-wind turbulent spectrum from MHD to electron scales, Phys. Rev. Lett.,
103, 165003, https://doi.org/10.1103/PhysRevLett.103.165003, 2009.

Alexandrova, O., Chen, C. H. K., Sorriso-Valvo, L., Horbury, T. S., and Bale, S. D.: Solar wind turbulence and the role of ion instabilities, Space Sci. Rev., 178, 101-139, https://doi.org/10.1007/s11214-013-0004-8, 2013.

Bale, S. D., Kellogg, P. J., Mozer, F. S., Horbury, T. S., and Rème, H.: Measurement of the electric fluctuation spectrum of magnetohydrodynamic turbulence, Phys. Rev. Lett., 94, 215002, https://doi.org/10.1103/PhysRevLett.94.215002, 2005.

Balogh, A. and Treumann, R. A.: Physics of Collisionless Shocks: Space Plasma Shock Waves, ISSI Scientific Reports Series Vol. 12, Springer, New York, 2013, Chap. 4, 149-220, 2013.

Barnsley, M.: Fractals Everywhere, Academic Press, Boston, 1988.

Baumjohann, W. and Treumann, R. A.: Basic Space Plasma Physics, Revised Edition 2012, Imperial College Press, London, 1996.

Biskamp, D.: Magnetohydrodynamic Turbulence, Cambridge University Press, Cambridge, 2003

Boldyrev, S. and Perez, J. C.: Spectrum of weak magnetohydrodynamic turbulence, Phys. Rev. Lett., 103, 225001, https://doi.org/10.1103/PhysRevLett.103.225001, 2009.

Brown, M. R., Schaffner, D. A. and Weck, P. J.: Magnetohydrodynamic turbulence: Observations and experiment, Phys. Plasmas., 22, 055601, https://doi.org/10.1063/1.4919391, 2015.

Celnikier, L. M., Harvey, C. C., Jegou, J., Moricet, P., and Kemp, M.: A determination of the electron density fluctuation spectrum in the solar wind, using the ISEE propagation experiment, Astron. Astrophys., 126, 293-298, 1983.

Chandran, B. D. G., Quataert, E., Howes, C., Xia, Q., and Pongkitiwanichakul, P.: Constraining low-frequency Alfvénic turbulence in the solar wind using density-fluctuation measurements, Astrophys. J., 707, 1668-1675, https://doi.org/10.1088/0004637X/707/2/1668, 2009.

Chaston, C. C., Bonnell, J. W., Carlson, C. W., McFadden, J. P., Ergun, R. E., and Strangeway, R. J.: Properties of small-scale Alfvén waves and accelerated electrons from FAST, J. Geophys. Res., 108, 8003, https://doi.org/10.1029/2002JA009420, 2003.

Chen, C. H. K., Bale, S. D., Salem, C., and Mozer, F. S.: Frame dependence of the electric field spectrum of solar wind turbulence, Astrophys. J. Lett., 737, 4 pp., https://doi.org/10.1088/20418205/737/2/L41, 2011.

Chen, C. H. K., Salem, C. S., Bonnell, J. W., Mozer, F. S., and Bale, S. D.: Density fluctuation spectrum of solar wind trubulence between ion and electron scales, Phys. Rev. Lett., 109, 035001, https://doi.org/10.1103/PhysRevLett.109.035001, 2012.

Eckmann, J. P. and Procaccia, I.: Fluctuations and dynamical scaling indices in nonlinear systems, Phys. Rev. Pt. A, 34, 659-661, https://doi.org/10.1103/PhysRevA.34.659, 1986.

Eckmann, J. P. and Ruelle, D.: Ergodic theory and strange attractors, Rev. Mod. Phys., 57, 617-656, https://doi.org/10.1103/RevModPhys.57.617, 1985.

Elsasser, W. M.: The hydromagnetic equations, Phys. Rev., 79, 183 183, https://doi.org/10.1103/PhysRev.79.183, 1950.

Goldstein, M. L., Roberts, D. A., and Matthaeus, W. H.: Magnetohydrodynamic turbulence in the solar wind, Ann. Rev. Astron. Astrophys., 33, 283-326, https://doi.org/10.1146/annurev.aa.33.090195.001435, 1995. 
Horbury, T. S., Wicks, R. T., and Chen, C. H. K.: Anisotropy in space plasma turbulence: solar wind observations, Space Sci. Rev., 172, 325-342, https://doi.org/10.1007/s11214-011-9821-9, 2012.

Kittel, C. and Kroemer, H.: Thermal Physics, W. H. Freeman Co., New York, Chap. 6, 179 pp., 1980.

Kolmogorov, A. N.: The local structure of turbulence in incompressible viscous fluid for very large Reynolds numbers, Dokl. Akad. Nauk SSSR 30, 299-303, 1941.

Kolmogorov, A. N.: A refinement of previous hypotheses concerning the local structure of turbulence in a viscous incompressible fluid at high Reynolds number, J. Fluid Mech., 13, 82-85, https://doi.org/10.1017/S0022112062000518, 1962.

Krall, N. A. and Trivelpiece, A. W.: Principles of Plasma Physics, McGraw-Hill, New York, 1973.

LaBelle, J. and Treumann, R. A.: Poynting vector measurements of electromagnetic ion cyclotron waves in the plasmasphere, J. Geophys. Res., 97, 13789-13797, https://doi.org/10.1029/92JA00990, 1992.

LaBelle, J. and Treumann, R. A.: Auroral radio emissions, 1. Hisses, roars, and bursts, Space Science Rev., 101, 295-440, https://doi.org/10.1023/A:1020850022070, 2002.

Landau, L. D., Lifshitz, E. M. and Pitaevskii, L. P.: Electrodynamics of Continuous Media, Butterworth-Heinemann, Oxford, 1998.

Landau, L. D. and Lifshitz, E. M.: Statistical Physics, Pt. I, Pergamon Press, Oxford, 130 pp., 1994.

Podesta, J. J., Roberts, D. A., and Goldstein, M. L.: Power spectrum of small-scale turbulent velocity fluctuations in the solar wind, J. Geophys. Res., 111, A10109, https://doi.org/10.1029/2006JA011834, 2006.

Podesta, J. J., Roberts, D. A., and Goldstein, M. L.: Spectral exponents of kinetic and magnetic energy spectra in solar wind turbulence, Astrophys. J., 664, 543-548, https://doi.org/10.1086/519211, 2007.

Podesta, J. J. and Borovsky, J. E.: Scale invariance of normalized cross-helicity throughout the inertial range of solar wind turbulence, Phys. Plasmas, 17, 112905, https://doi.org/10.1063/1.3505092, 2010.

Podesta, J. J.: Spatial scales and temporal scales in the theory of magnetohydrodynamic turbulence, Phys. Plasmas, 18, 012906, https://doi.org/10.1063/1.3534824, 2011a.

Podesta, J. J.: On the cross-helicity dependence of the energy spectrum in magnetohydrodynamic turbulence, Phys. Plasmas, 18, 012907, https://doi.org/10.1063/1.3533671, 2011 b.

Roberts, D. A.: Evolution of the spectrum of solar wind velocity fluctuations from 0.3 to 5 AU, J. Geophys. Res., 115, A12101, https://doi.org/10.1029/2009JA015120, 2010.

Šafránková, J., Nemeček, Z., Přech, L., and Zastenker, G. N.: Ion kinetic scale in the solar wind observed, Phys. Rev. Lett., 110, 25004, https://doi.org/10.1103/PhysRevLett.110.025004, 2013.

Šafránková, J., Nemeček, Z., Němec, F., Přech, L., Chen, C. H. K., and Zastenker, G. N.: Power spectral density of fluctuations of bulk and thermal speeds in the solar wind, Astrophys. J., 825, 8 pp., https://doi.org/10.3847/0004-637X/825/2/121, 2016.

Sahraoui, F., Goldstein, M. L., Belmont, G., Canu, P., and Rezeau, L.: Evidence of a cascade and dissipation of solar wind turbulence at the electron gyroscale, Phys. Rev. Lett., 102, 231102 , https://doi.org/10.1103/PhysRevLett.102.231102, 2009.
Sahraoui, F., Belmont, G., and Goldstein, M. L.: New insight into short-wavelength solar wind fluctuations from Vlasov theory, Astrophys. J., 748, 11 pp., https://doi.org/10.1088/0004637X/748/2/100, 2012.

Sahraoui, F., Huang, S. Y., Belmont, G., Goldstein, M. L., Retinò, A., Robert, P., and De Patoul, J.: Scaling of the electron dissipation range of solar wind turbulence, Astrophys. J. Lett., 777, 11 pp., https://doi.org/10.1088/0004-637X/777/1/15, 2013.

Stawarz, J. E., Eastwood, J. P., Varsani, A., Ergun, R. E., Shay, M. A., Nakamura, R., Phan, T. D., Burch, J. L., Gershman, D. J., Giles, B. L., Goodrich, K. A., Khotyaintsev, Y. V., Lindqvist, P.A., Russell, C. T., Strangeway, R. J., and Torbert, R. B.: Magnetospheric multiscale analysis of intense field-aligned Poynting flux near the Earth's plasma sheet boundary, Geophys. Res. Lett., 44, 7106-7113, https://doi.org/10.1002/2017GL073685, 2017.

Sundqvist, D., Krasnoselskikh, V., Bale, S. D., Schwartz, S. J., Soucek, J., and Mozer, F.: Dispersive nature of high Mach number collisionless plasma shocks: Poynting flux of oblique whistler waves, Phys. Rev. Lett., 108, 025002, https://doi.org/10.1103/PhysRevLett.108.025002, 2012.

Treumann, R. A., Baumjohann, W., and Narita, Y.: Ideal MHD turbulence: The inertial range spectrum with collisionless dissipation, Front. Phys., 3, 6 pp., https://doi.org/10.3389/fphys.2015.00022, 2015.

Treumann, R. A. and Baumjohann, W.: Spontaneous magnetic reconnection. Collisionless reconnection and its potential astrophysical relevance, Astron. Astrophys. Rev., 23, 91 pp., https://doi.org/10.1007/s00159-015-0087-1, 2015.

Treumann, R. A. and Baumjohann, W.: Inverse scattering problem in turbulent magnetic fluctuations, Ann. Geophys., 34, 673-689, https://doi.org/10.5194/angeo-34-673-2016, 2016.

Treumann, R. A. and Baumjohann, W.: Poynting's theorem in magnetic turbulence, accessible at arXiv:1701.01266v3 (physics.space-ph), 2017.

Treumann, R. A. and Baumjohann, W.: Electron cyclotron maser instability (ECMI) in strong magnetic guide field reconnection, Ann. Geophys., 35, 999-1013, https://doi.org/10.5194/angeo35-999-2017, 2017.

Wicks, R. T., Forman, M. A., Horbury, T. S., and Oughton, S.: Power anisotropy in the magnetic field power spectral tensor of solar wind turbulence, Astrophys. J., 746, 103-126, https://doi.org/10.1088/0004-637X/746/1/103, 2012.

Yoon, P. H.: Kinetic theory of hydromagnetic turbulence, I. Formal results for parallel propagation, Phys. Plasmas, 14, 102302 , https://doi.org/10.1063/1.2780139, 2007.

Yoon, P. H. and Fang, T. M.: Kinetic theory of hydromagnetic turbulence, II. Susceptibilities, Phys. Plasmas, 14, 102303, https://doi.org/10.1063/1.2780140, 2007.

Zank, G. P., Dosch, A., Hunana, P., Florinski, V., Matthaeus, W. H., and Webb, G. M.: The transport of low-frequency turbulence in astrophysical flows, I. Governing equations, Astrophys. J., 745, 20 pp., https://doi.org/10.1088/X/745/1/35, 2012.

Zaslavsky, G. M.: Chaos in Dynamic Systems, Harwood, Chur, 1985.

Zhou, Y., Matthaeus, W. H., and Dmitruk, P.: Magnetohydrodynamic turbulence and time scales in astrophysical and space plasmas, Rev. Mod. Phys., 76, 1015-1035, https://doi.org/10.1103/RevModPhys.76.1015, 2004. 